\title{
A SYMMETRY ANALYSIS OF ELECTRIC-FIELD-INDUCED SPECTRA
}

\author{
David L. ANDREWS and B.S. SHERBORNE \\ School of Chemical Sciences, University of East Anglia, Nonwich NRA 7TJ, UK
}

Received 21 February 1984

\begin{abstract}
The application of an electric field is shown to allow access to previousiy unobserved vibronic states when the perturbed molecules are probed by a laser. The appropriate absorption rate is calculated using standard quantum electrodynamics, in the electric-dipole approximation. Random orientation of the molecules is assumed, corresponding to the case where the molecules have no permanent dipole moment. Reduction of the resulting expression to its irreducible, second-rank tensor components allows selection rules for this electric-field-induced spectroscopy (EFIS) to be derived. These are similar to those for the two-photon spectroscopies, such as Raman scattering and two-photon absorption. Variation of the angle between the two fields is shown to give definite identification of the transition symmetry in most cases.
\end{abstract}

\section{Introduction}

It is well known that an electric field can perturb the energies of atoms and molecules in a process termed the Stark effect. An applied electric field also generally induces a mixing of the eigenstates of the system, resulting in a change of the selection rules. Such an effect is manifested by the observation of fundamental vibration-rotation features in the spectra of homonuclear diatomics such as $\mathrm{H}_{2}$ [1]. The appearance of field-induced absorption lines was predicted by Condon [2], who drew attention to the analogy with Raman spectroscopy, in the limit where one photon has zero frequency. Raman-like selection rules have recently been observed in the electric-field-induced spectrum of ammonia [3].

In this paper, electric-field-induced spectroscopy (EFIS) is considered within the framework of quantum electrodynamics, with the interactions considered in the electric-dipole approximation. The problem is simplified by assuming the molecules to be randomly oriented, which corresponds to a fluid phase in which the molecules have no permanent electric-dipole moment. Analysis of the EFIS absorption rate as a function of the angle between the static and electromagnetic fields yields similar information to a double-beam, two-photon experiment. Thus it is shown that not only can EFIS access new states, as in two-photon absorption studies, but that it also gives an unequivocal assignment of the transition symmetry in most cases.

\section{Theory}

The molecular rate of single-photon absorption given by the standard methods of time-dependent perturbation theory, is as follows

$\Gamma=\left(\pi I / \epsilon_{0} \hbar c\right)\left\langle\left|\mu^{f 0} \cdot e\right|^{2}\right\rangle \rho_{\mathrm{f}}$.

Here, $I$ is the irradiance and $e$, the unit polarisation vector of the radiation; $\mu^{f 0}$ is the transition dipole moment for the optical transition, $|f\rangle \leftarrow$ $|0\rangle ; \rho_{\mathrm{r}}$, the density of final states, and the angular brackets denote rotational averaging to account for the random orientation of the molecules in a fluid.

In this paper, transitions are considered which are electric-dipole (E1) forbidden:

$\mu^{f 0}=0$,

but which may be induced by an applied electric field. The appropriate wavefunctions, $\left|0^{\prime}\right\rangle$ and $\left|f^{\prime}\right\rangle$, 
are thus given by time-independent perturbation theory:

$$
\begin{aligned}
& \left|0^{\prime}\right\rangle=|0\rangle+\sum_{r \neq 0}\left(\mu^{r 0} \cdot \mathscr{E}\right) E_{0 r}^{-1}|r\rangle+\ldots, \\
& \left|f^{\prime}\right\rangle=|f\rangle+\sum_{r \neq f}\left(\mu^{r f} \cdot \mathscr{E}\right) E_{f r}^{-1}|r\rangle+\ldots,
\end{aligned}
$$

where $E_{s r}$ is the difference in the zeroth-order energies, $E_{s}-E_{r}(s=f$ or 0$)$. The corresponding EFIS absorption rate, from eq. (2.1), is then

$$
\begin{aligned}
\Gamma= & \left(\pi I / \epsilon_{0} \hbar c\right)\langle| \sum_{r \neq f}\left(\mu^{f r} \cdot \mathscr{E}\right)\left(\mu^{r 0} \cdot e\right) /\left(E_{0 r}+\hbar \omega\right) \\
& \left.+\sum_{r \neq 0}\left(\mu^{f r}-e\right)\left(\mu^{r 0} \cdot \mathscr{E}\right) /\left.E_{0 r}\right|^{2}\right\rangle \rho_{\mathrm{f}},
\end{aligned}
$$

where use has been made of the energy conservation equation

$E_{f}=E_{0}+\hbar \omega$.

A more direct approach to eq. (2.5) is through use of standard electrodynamical methods [4]. using as the perturbation operator

$\mathscr{H}_{\text {int }}=-\epsilon_{0}^{-1} \mu \cdot d^{\perp}-\mu \cdot \mathscr{E}$,

where $\mu$ is the electric-dipole moment operator, and $d^{\perp}$, the transverse electric-displacement operator for the radiation field. In this case. the rate expression is derived from the Fermi rule

$\Gamma=(2 \pi / \hbar)\left\langle\left|M_{f 0}\right|^{2}\right\rangle \rho_{\mathrm{f}}$,

using the second term in the expansion for $M_{f 0}$ :

$$
\begin{aligned}
M_{f 0}= & \left\langle f_{s}\left|\mathscr{H}_{\text {int }}\right| 0_{s}\right\rangle \\
& +\sum_{r_{s}} \frac{\left\langle f_{s}\left|\mathscr{H}_{\text {int }}\right| r_{s}\right\rangle\left\langle r_{s}\left|\mathscr{H}_{\text {int }}\right| 0_{s}\right\rangle}{E_{0_{s}}-E_{r_{s}}} .
\end{aligned}
$$

The subscript, $s$, denotes that the states refer to states of the system comprising the molecule and the radiation. The two terms appearing in eq. (2.5), may now be associated with time-ordered diagrams, as shown in fig. 1.

To proceed further, it is noted that by virtue of eq. (2.2), each summation over the virtual states, $|r\rangle$, in eq. (2.5) excludes both the ground state and the final state. The result may be expressed in terms of a real molecular response tensor, $S_{i j}$,

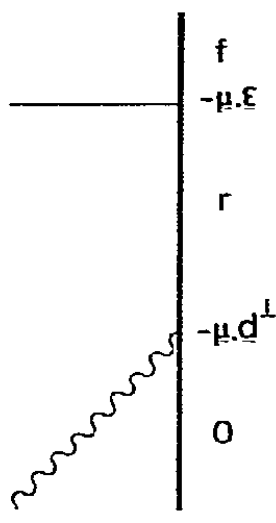

(a)

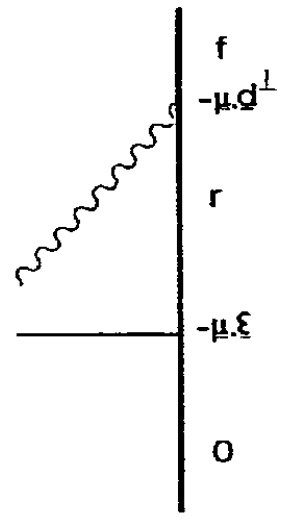

(b)
Fig. 1. Time-ordered diagrams for electric-field-induced spectroscopy.

defined as

$S_{t j}=\sum_{r}^{\prime}\left[\mu_{a}^{f r} \mu_{j}^{r 0} /\left(E_{0 r}+\hbar \omega\right)+\mu_{J}^{f r} \mu_{a}^{r 0} /\left(E_{0 r}\right)\right]$,

where the prime on the summation denotes, $r \neq 0$, $f$. The rate equation is then:

$\Gamma_{\mathrm{EFIS}}=\left(\pi I / \epsilon_{0} \hbar c\right)\left\langle\left|S_{i j} \mathscr{E}_{\imath} e_{l}\right|^{2}\right\rangle \rho_{\mathrm{f}}$,

where use has been made of the implied summation convention for repeated indices.

The required rotational average may be effected by first referring the molecular tensor components to a molecule-fixed frame, denoted by Greek indices, in which they are rotationally invariant.

$$
\begin{aligned}
\Gamma_{\mathrm{EFIS}}= & \left(\pi I \rho_{\mathrm{f}} / \epsilon_{\mathrm{o}} \hbar c\right) S_{\lambda \mu} S_{\nu \mathrm{o}} \mathscr{E}_{2} e_{j} \mathscr{E}_{k} \bar{e}_{I} \\
& \times\left\langle l_{i \lambda} l_{j \mu} l_{k \nu} l_{l \mathrm{o}}\right\rangle .
\end{aligned}
$$

In eq. (2.12), the direction cosine $l_{i \lambda}$, for example denotes the $(i, \lambda)$ element of the Euler angle matrix [5] for the transformation between the two frames. The rotational average of the product of the direction cosines in eq. (2.12), is then a standard result [6]:

$$
\left\langle l_{i \lambda} l_{j \mu} l_{k-v} l_{l o}\right\rangle=\frac{1}{30}\left[\begin{array}{ll}
\delta_{i j} & \delta_{k l} \\
\delta_{i k} & \delta_{j l} \\
\delta_{i l} & \delta_{j k}
\end{array}\right]^{\mathrm{r}}
$$




$$
\times\left[\begin{array}{rrr}
4 & -1 & -1 \\
-1 & 4 & -1 \\
-1 & -1 & 4
\end{array}\right]\left[\begin{array}{ll}
\delta_{\lambda \mu} & \delta_{\nu 0} \\
\delta_{\lambda \nu} & \delta_{\mu 0} \\
\delta_{\lambda 0} & -\delta_{\mu \nu}
\end{array}\right]
$$

leading to the following expression for the absorption rate:

$$
\begin{aligned}
\Gamma_{\mathrm{EFIS}}= & \left(\pi I \mathscr{E}^{2} \rho_{\mathrm{f}} / 30 \hbar c \epsilon_{0}\right) \\
& \times\left[\left(3 S_{\lambda \lambda} S_{\mu \mu}+3 S_{\lambda \mu} S_{\mu \lambda}-2 S_{\lambda \mu} S_{\lambda \mu}\right) \cos ^{2} \psi\right. \\
& \left.+\left(4 S_{\lambda \mu} S_{\lambda \mu}-S_{\lambda \lambda} S_{\mu \mu}-S_{\lambda \mu} S_{\mu \lambda}\right)\right],
\end{aligned}
$$

where

$|\mathscr{E} \cdot e|^{2}=\mathscr{E}^{2} \cos ^{2} \psi$

In the case of plane-polarised light $\psi$ simply represents the angle between the electric field vector and the polarisation vector.

The most effective method of dealing with the selection rules for EFIS, is to introduce irreducible tensors. The decomposition of a second-rank cartesian tensor into its irreducible parts takes the form

$S_{\lambda \mu}=S_{\lambda \mu}^{(0)}+S_{\lambda \mu}^{(1)}+S_{\lambda \mu}^{(2)}$,

$S_{\lambda \mu}^{(0)}=\frac{l}{3} \delta_{\lambda \mu} S_{\nu \nu}$,

$S_{\lambda \mu}^{(1)}=\frac{1}{2}\left(S_{\lambda \mu}-S_{\mu \lambda}\right)$,

$S_{\lambda \mu}^{(2)}=\frac{1}{2}\left(S_{\lambda \mu}+S_{\mu \lambda}\right)-\frac{1}{3} \delta_{\lambda \mu} S_{\nu \bar{y}}$,

Here, $S_{\lambda \mu}^{(0)}$ is a weight 0 tensor which transforms under the operations of the full rotation group as a scalar; $S_{\lambda \mu}^{(1)}$ is a weight 1 tensor, which transforms as a pseudo-vector (i.e. its components have the same transformation properties as the rotations, $\left.R_{x}, R_{y}, R_{z}\right)$; and $S_{\lambda \mu}^{(2)}$ is a weight-2 tensor which transforms as a second-rank, symmetric, traceless tensor (i.e. its components transform in the same way as $x y, x z, y z, x^{2}-y^{2}$ and $2 z^{2}-x^{2}-y^{2}$ ).

The three molecular tensor products in eq. (2.14), may now be expressed in terms of the three irreducible tensor products $S_{\lambda_{\mu}}^{(0)} S_{\lambda_{\mu}}^{(0)}, S_{\lambda \mu}^{(1)} S_{\lambda \mu}^{(1)}$ and $S_{\lambda_{\mu}}^{(2)} S_{\lambda_{\mu}}^{(2)}$, each of which is real and positive:

$S_{\lambda \lambda} S_{\mu \mu}=3 S_{\lambda \mu}^{(0)} S_{\lambda \mu}^{(0)}$,

$S_{\lambda \mu} S_{\lambda \mu}=S_{\lambda \mu}^{(0)} S_{\lambda \mu}^{(0)}+S_{\lambda \mu}^{(1)} S_{\lambda \mu}^{(1)}+S_{\lambda \mu}^{(2)} S_{\lambda \mu}^{(2)}$,

$$
S_{\lambda \mu} S_{\mu \lambda}=S_{\lambda \mu}^{(0)} S_{\lambda \mu}^{(0)}-S_{\lambda \mu}^{(1)} S_{\lambda \mu}^{(1)}+S_{\lambda \mu}^{(2)} S_{\lambda \mu}^{(2)}
$$

Substitution of these expressions in eq. (2.14) leads to;

$$
\begin{aligned}
\Gamma_{\mathrm{EFIS}}= & \left(\pi I \mathscr{E}^{2} \rho_{\mathrm{f}} / 30 \hbar c \epsilon_{0}\right) \\
& \times\left[\left(10 S_{\lambda \mu}^{(0)} S_{\lambda \mu}^{(0)}-5 S_{\lambda \mu}^{(1)} S_{\lambda \mu}^{(1)}+S_{\lambda \mu}^{(2)} S_{\lambda \mu}^{(2)}\right) \cos ^{2} \psi\right. \\
& \left.+\left(5 S_{\lambda \mu}^{(1)} S_{\lambda \mu}^{(1)}+3 S_{\lambda \mu}^{(2)} S_{\lambda \mu}^{(2)}\right)\right] .
\end{aligned}
$$

The dependence of the absorption rate on the experimentally controllable parameter $\psi$ may thus be expressed through the relation

$\Gamma_{\mathrm{EFIS}}(\psi) \propto 1+\alpha \cos ^{2} \psi$,

where

$\alpha=\frac{10 S_{\lambda \mu}^{(0)} S_{\lambda \mu}^{(0)}-5 S_{\lambda \mu}^{(1)} S_{\lambda \mu}^{(1)}+S_{\lambda \mu}^{(2)} S_{\lambda \mu}^{(2)}}{5 S_{\lambda \mu}^{(1)} S_{\lambda \mu}^{(1)}+S_{\lambda \mu}^{(2)} S_{\lambda \mu}^{(2)}}$,

the value of which should be directly measurable from a study of the $\psi$ dependence, using eq. (2.24).

\section{Selection rules}

For a field-induced transition originating from a totally symmetric ground state, the symmetry of the final state dictates the representation under which components of the molecular tensor must transform. In this section, application of this selection rule to the result derived in section 2 is considered. In other cases, where the molecular ground state is not totally symmetric, similar arguments apply: here it is the product of the initial and final state representations which dictates the transformation properties of the active tensor components. In general, each irreducible tensor may have zero, or non-zero components according to the symmetry of the final state. There are six possible cases, as shown in table 1; the case in which weights 0 and 1 alone are allowed does not arise $*$.

* If weight 0 is allowed then the final state must belong to the totally symmetric representation; if weight 1 is also allowed under this representation, then its product with itself must transform similarly: thus, components of weight $\mathbf{2}$ must also then transform under the same totally symmetric representation. 
Table 1

Values of the experimental parameter $a$ for the six possible transition symmetries

\begin{tabular}{llc}
\hline Case & Allowed weights & $\alpha$ \\
\hline (a) & $0,1,2$ & $>-1$ \\
(b) & 1,2 & $-1<\alpha<\frac{1}{3}$ \\
(c) & 0,2 & $>\frac{1}{3}$ \\
(d) & 2 & $\frac{1}{3}$ \\
(e) & 1 & -1 \\
(f) & 0 & $\infty$ \\
\hline
\end{tabular}

To illustrate some of these cases, we consider EFIS in a molecule of $D_{3 h}$ symmetry, such as $s$-triazine which has been studied recently by Lombardi and Scheps [7]. Reference to the appropriate character table shows that there are two El-allowed transitions $\left(A_{1}^{\prime} \rightarrow A_{2}^{\prime \prime} ; A_{1}^{\prime} \rightarrow E^{\prime}\right)$, while there are three that may be field induced $\left(A_{1}^{\prime} \rightarrow A_{1}^{\prime}\right.$; $\left.A_{1}^{\prime} \rightarrow A_{2}^{\prime}: A_{1}^{\prime} \rightarrow E^{\prime \prime}\right)$. In the first of these, $A_{1}^{\prime} \rightarrow A_{1}^{\prime}$. weights 0 and 2 are permitted but weight 1 components are not; hence according to table 1 , we have $\alpha>\frac{1}{3}$. For a transition, $A_{1}^{\prime} \rightarrow A_{2}^{\prime}$, weight 1 alone is permitted, and hence $\alpha=-1$. Finally, for a transition $A_{1}^{\prime} \rightarrow E^{\prime \prime}$, weights 1 and 2 alone are permitted and hence $\alpha$ lies in the range, $-1<\alpha<\frac{1}{3}$.

The above example demonstrates one of the many cases in which an experimental determination of the parameter $\alpha$ provides for an unequivocal characterisation of the excited state symmetry for a field-induced transition. In general, use may be made of the results of table 1 , cast into diagrammatic form, as in fig. 2 . There appears to be the possibility of ambiguity, for certain values of $\alpha$, in distinguishing between cases (a) and (b), or between cases (a) and (c). However, cases (a) and (c) cannot arise together in any point group, since there is only one totally symmetric representation; also, case (a) can only occur in molecules belonging to the point groups, $\mathrm{C}_{n \mathrm{~h}}$ or $\mathrm{S}_{n}$ (assuming the transition is E1 forbidden). Hence, in almost all applications, experimental determination of $\alpha$ provides unambiguous symmetry information on each field-induced spectral feature.

One other point of interest concerns EFIS for which only the weight 0 tensor components are non-zero, case (f). Such induced transitions belong to totally symmetric representations, and only occur in molecules belonging to the cubic, or icosohedral point groups. First it is noted that such transitions are not only E1, but also $\mathrm{M} 1$ and E2

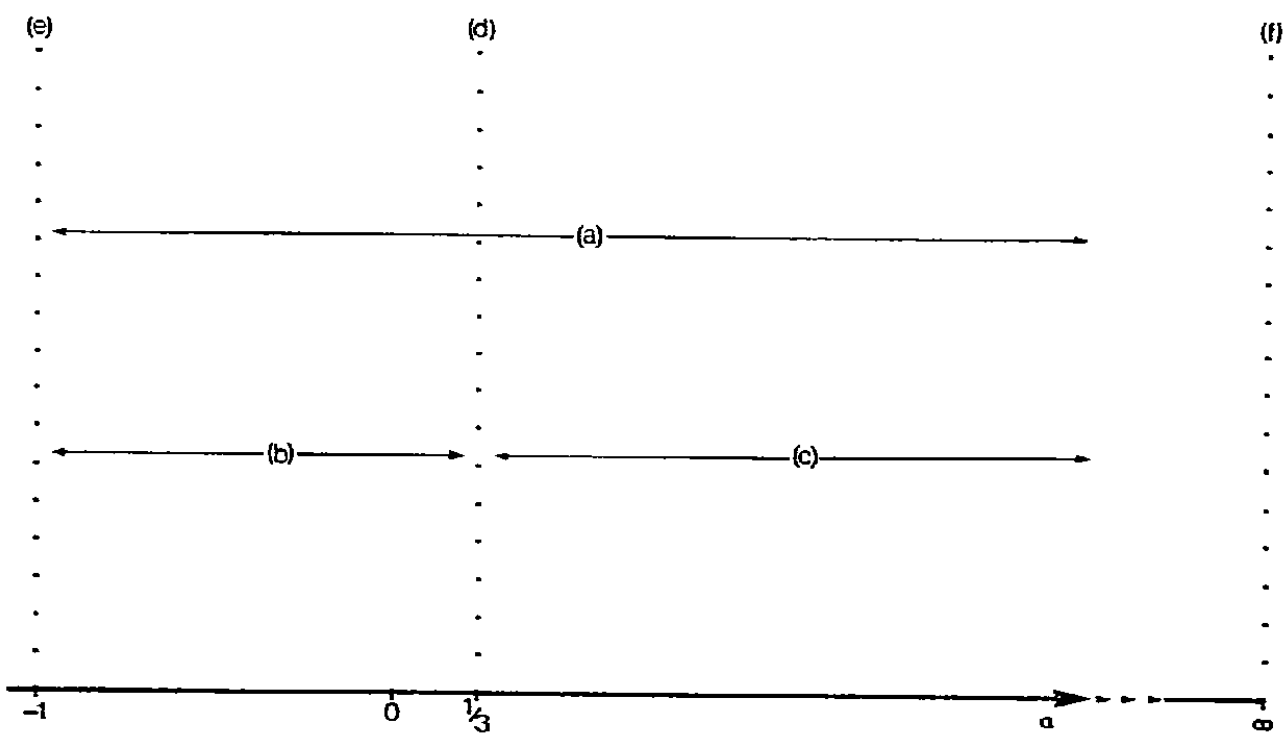

Fig. 2. Classification of electric-field-induced transitions according to the value of $\alpha$. 
forbidden ${ }^{*}$, and hence cannot even weakly occur in the absence of the electric field. Secondly, the result $\alpha=\infty$ implies that dependence of the $a b$ sorption rate, eq. (2.23), has been reduced to a simple $\cos ^{2} \psi$ dependence; this feature should again be directly amenable to experimental verification.

\section{Discussion}

It is interesting to compare the EFIS process, with double-beam, two-photon absorption. For two-photon absorption, we have

$\Gamma_{\mathrm{TPA}}=\left(\pi I^{\prime} / 2 \hbar c^{2} \epsilon_{\mathrm{o}}\right)\left\langle\left|T_{i j} e_{i}^{\prime} e_{j}\right|^{2}\right\rangle \rho_{\mathrm{f}}$,

where $e^{(1)}$, is the polarisation of the beam with intensity $I^{(\prime)}$, and $E_{f o}=\hbar \omega+\hbar \omega^{\prime}$. The field-induced single-photon absorption rate, eq. (2.11), may be similarly expressed as

$\Gamma_{\mathrm{EFIS}-}\left(\pi I \mathscr{E}^{2} / \hbar c \epsilon_{0}\right)\left\langle\left|S_{i j} \hat{\mathscr{E}}_{i} e_{j}\right|^{2}\right\rangle \rho_{\mathrm{r}}$.

The two-photon absorption tensor, $T_{i j}$, differs from $S_{1}$, only by the addition of $\hbar \omega^{\prime}$, in the denominator of the second term in eq. (2.10). Hence, $S_{i j}$ may be regarded as the low-frequency limit $\left(\omega^{\prime} \rightarrow 0\right)$ of $T_{i j}$.

Comparison of eqs. (4.1) and (4.2) enables an estimation of the relative likelihood of observing the field-induced process. Given the similarity of the molecular tensors, the ratio of the two rates is approximately given by

$\Gamma_{\mathrm{EFIS}} / \Gamma_{\mathrm{TPA}} \approx 2 \mathscr{E}^{2} c \epsilon_{0} / I^{\prime}$.

For a pulsed laser irradiance of $10^{13} \mathrm{~W} \mathrm{~m}^{-2}$,

* M1 (magnetic-dipole) transitions have selection rules governed by the transformation properties of the pseudovectors (weight 1), while E2 (electric-quadrupole) transitions are associated with traceless, symmetric, second-rank tensors (weight 2). which is typical for use in two-photon studies, and an experimentally. feasible $[8,9]$ electric-field strength of $5 \times 10^{7} \mathrm{~V} \cdot \mathrm{m}^{-1}$, the ratio is approximately unity. The viability of EFIS is further increased, if one considers its quadratic dependence on the applied field. Like the Stark effect utilised in the observation of molecular rotation spectra, ac fields coupled with phase sensitive detection serves to separate any induced features from the standard absorption spectrum, and generally increase the signal-to-noise ratio.

The possibility of using a static electric field to change the selection rules for a transition has recently been considered as a method of "dumping" energy stored in a metastable state [10]. Hopefully the symmetry considerations that have been outlined here may help the choice of suitable candidates for such gain switches.

\section{Acknowledgement}

One of us BSS gratefully acknowledges financial support from the Science and Engineering Research Council.

\section{References}

[1] R.W. Terhune and C.W. Peters, J. Mol. Spectry. 3 (1959) 138.

[2] E.U. Condon, Phys. Rev. 41 (1932) 759.

[3] W.H. Weber and E.A. Cohen, Opt. Letters 8 (:983) 488.

[4] D.P. Craig and T. Thirunamachandran, Advan. Quantum. Chem. 16 (1982) 97.

[5] J.L. Synge and B.A. Griffith, in: Principles of mechanics (McGraw-Hill, New York, 1959).

[6] D.L. Andrews and T. Thirunamachandran, J. Chem. Phys. 67 (1977) 5026.

[7] J.R. Lombardi and R. Scheps, Chem. Phys. 10 (1975) 445.

[8] W. Liptay, in: Excited states, ed. E.C. Lim (Academic Press, New York, 1974).

[9] Y. Amako, Y. Sato and A. Watanabe, Bull. Chem. Soc. Japan 51 (1978) 360.

[10] K. Drühl, M.O. Scully. and A.W. Overhauser, Opt. Commun. 38 (1981) 393. 